\title{
Hypertrophic Scar
}

National Cancer Institute

\section{Source}

National Cancer Institute. Hypertrophic Scar. NCI Thesaurus. Code C26997.

A permanent mark on the skin caused by deposits of excessive amounts of collagen

during wound healing. It is elevated and does not extend beyond the original boundaries

of the wound; the elevation may stabilize or regress spontaneously. 